This is a post-peer-review, pre-copyedit version of an article published in Der Schmerz. The final authenticated version is available online at: https://doi.org/10.1007/s00482-018-0329-z.

Originalien

N. Niederstrasser 1, B. Steiger ${ }^{2}$, K. Welsch ${ }^{3}$, S. Hartmann ${ }^{4}$, P. Nilges ${ }^{5}$, A. Ljutow ${ }^{6}$, D. Ettlin ${ }^{2}$

1) Faculty of Health and Life Sciences, De Montfort University, The Gateway, Leicester, United Kingdom

2) Interdisziplinäre orofaziale Schmerzsprechstunde, Zentrum für Zahnmedizin (ZZM), Universität Zürich , Schweiz

3) Schmerzambulanz der Klinik für Anästhesiologie, Intensivmedizin und Schmerztherapie, Universität des Saarlandes Homburg (UKS), Deutschland

4) Schmerzzentrum, Kantonsspital St. Gallen (KSG), Schweiz

5) DRK Schmerzzentrum, Mainz (DRK), Deutschland

6) Zentrum für Schmerzmedizin, Schweizerisches Paraplegiker-Zentrum Nottwil (SPZ), Schweiz

\title{
Deutsche transkulturelle Übersetzung des Injustice Experience Questionnaire (IEQ)
}

Korrespondenzadresse

Dr. Nils Georg Niederstrasser

Faculty of Health and Life Sciences

De Montfort University

The Gateway, Leicester

LE1 9BH, United Kingdom

tel: $+44(0) 1163664365$

nils.niederstrasser@dmu.ac.uk

\section{Zusatzmaterial online}

Die Online-Version dieses Beitrags (doi: 10.1007/s00482-018-0329-z) enthält weitere Texte verschiedene Reports - zu den Übersetzungen.

Beitrag und Zusatzmaterial stehen Ihnen im elektronischen Volltextarchiv auf http://www.springermedizin.de/der-schmerz zur Verfügung. Sie finden das Zusatzmaterial am Beitragsende unter „Supplementary Material“. 


\title{
Zusammenfassung
}

Einleitung: Die berufliche und soziale Rehabilitation wird durch wahrgenommene Ungerechtigkeit infolge von Verletzungen beeinflusst. Zu deren Einschätzung ist der Injustice Experience Questionnaire (IEQ) auf Englisch verfügbar. Ziel dieser Studie war die deutschsprachige transkulturelle Übersetzung.

Methodik: Der IEQ wurde nach den Kriterien zur transkulturellen Anpassung von Selbsteinschätzungs-Instrumenten ins Deutsche übersetzt. An einer Stichprobe von 19 Schmerzpatienten wurde untersucht, ob die übersetzten Items unverständlich, inakzeptabel oder anstössig waren, wie auch was deren Bedeutung und der Grund für die gewählte Antwortoption war. Die Auswertung der Daten erfolgte mit nichtparametrischen statistischen Verfahren.

Resultate: Die deutsche Übersetzung des IEQ zeigte ein hohes Maß an Verständlichkeit. Die Bedeutung der Items und die gewählten Antwortoptionen wurden von zwei Ratern in hohem Ausmaß als plausibel eingestuft und die Formulierung der Items von den Teilnehmenden weder als inakzeptabel noch als beleidigend bewertet. Aufgrund leicht erhöhter Werte bei der nicht erkannten Bedeutung durch die Rater bei Item 3 wurde in diesem der Begriff "Unachtsamkeit" durch "Unaufmerksamkeit" ersetzt.

Schlussfolgerung: Die Studie attestiert der Übersetzung des IEQs gute Verständlichkeit und Präzision. In einer Folgestudie sollte die Übersetzung an einer größeren Stichprobe Schmerzpatienten validiert werden.

\section{Schlüsselwörter:}

Ungerechtigkeit, Trauma, Schmerz, Fragebogen, Übersetzung

\begin{abstract}
Introduction: Occupational and social rehabilitation is influenced by perceived injustice as a result of injury. To assess perceived injustice, the Injustice Experience Questionnaire (IEQ) has been developed and is available in English. The aim of this study was to translate and culturally adapt the English version of the IEQ into German.

Methodology: The IEQ was translated and adapted into German according to the criteria for transcultural adaptation of self-assessment tools. The translation was examined in a sample of 19 pain patients for its comprehensibility and item meanings, as well as offensiveness. Data were assessed using nonparametric statistical methods.

Results: The German translation of the IEQ showed a high degree of comprehensibility. The items' meanings and participants' selected answer options were rated as highly plausible by two raters. Item wordings were rated neither as offensive nor unacceptable by participants. The German translation of the english term "negligence" in item 3 by the term "Unachtsamkeit" was assessed as misunderstandable, therefore it was replaced by the term "Unaufmerksamkeit".
\end{abstract}


Conclusion: The study attests the cultural and linguistic intelligibility and precision of the German translation of the IEQ. In a follow-up study, the translation should be validated in a larger sample of pain patients.

\section{Keywords:}

Injustice, trauma, pain, questionnaire, translation

\section{Einleitung}

Verletzungen mit nachhaltigen Schäden werden häufig als bedeutende Verluste charakterisiert. Letztere können körperliche und psychische Funktionen, Berufstätigkeit, Genussfähigkeit, finanzielle Sicherheit und Unabhängigkeit limitieren $[3,15]$. Solche Verlusterfahrungen werden oft als unverdient und die dadurch veränderten Lebensumstände als ungerecht wahrgenommen, insbesondere wenn die Verletzung durch Nachlässigkeit oder Fehler anderer erfolgte [6].

Grundsätzlich werden Situationen und Lebenserfahrungen dann als ungerecht bewertet, wenn diese durch eine Verletzung grundlegender Menschenrechte sowie Übertretungen betreffend Status oder Rang gekennzeichnet sind, oder wenn finanzielle Gerechtigkeit oder wichtige persönliche Überzeugungen in Frage gestellt werden [2,4,7].

Das meistgenutzte Instrument zur Auswertung wahrgenommener Ungerechtigkeit nach Verletzungen ist der Injustice Experience Questionnaire (IEQ), der an einer Population von Patienten mit muskuloskelettalen Beschwerden als Folge eines Unfalls entwickelt und validiert wurde [12]. Die Autoren des IEQ konzipierten wahrgenommene Ungerechtigkeit als 'an appraisal cognition comprising elements of the severity of loss consequent to injury, blame, a sense of unfairness, and irreparability of loss' [12] .

Der IEQ reflektiert verschiedene Aspekte von wahrgenommener Ungerechtigkeit wie Schädigung durch andere, irreparabler Verlust der bisherigen Lebensqualität oder eingeschränkte Entwicklungsmöglichkeiten in der Zukunft. Respondenten bewerten die Häufigkeit des Auftretens von auf wahrgenommene Ungerechtigkeit bezogener Gedanken und Gefühle [12].

In der bisherigen Forschung mit Patientengruppen mit heterogenen Schmerzursachen und posttraumatischen Stresssymptomen bewährte sich der IEQ als spezifischer Prädiktor für verlangsamte Rehabilitation und problematische Schmerzfolgen $[5,8,10,13,14,17]$.

Für die klinische Praxis und Forschung liegen bisher validierte Übersetzungen des IEQ in dänischer [5], französischer [12], japanischer [18] und spanischer Sprache [8] vor. Die Nutzung des IEQ in deutschsprachigen Populationen erfordert deshalb eine transkulturelle Übersetzung sowie deren Validierung, um semantische, linguistische und kulturelle Äquivalenz zu gewährleisten [1].

Ziel dieser Studie war die transkulturelle Anpassung und Übersetzung des IEQ in die deutsche Sprache, sowie Vortestung einer präfinalen deutschen Version an einer Stichprobe von Schmerzpatienten. Hierbei wurde vor allem auf untersucht ob die Übersetzung verständlich ist, von Patienten akzeptiert wird und nicht beleidigend ist.

\section{Material und Methoden}

Die englische Version des IEQ wurde nach den Kriterien zur transkulturellen Anpassung von Selbsteinschätzungs-Instrumenten ins Deutsche übersetzt [1]. Die für jede Phase erstellten Berichte sind im Anhang zu finden. 
In der ersten Phase erstellten zwei unabhängige Mitarbeitende mit deutscher Muttersprache eine deutsche Übersetzungen des IEQ, nämlich die Versionen T1 und T2 (T: Translation). Übersetzer 1 war sich der untersuchten Konzepte des Fragebogens bewusst, während Übersetzer 2 darüber nicht informiert war und keinerlei klinischen Hintergrund besaß.

\section{Phase 2: Synthese der Versionen T1 und T2}

Die Übersetzungen T1 und T2 (ESM 1 und ESM2 online) wurden in der Anwesenheit von Übersetzer 1 und 2 sowie eines Protokollführers miteinander verglichen. Semantische und begriffliche Diskrepanzen, welche während des Übersetzungsprozesses aufkamen, wurden identifiziert und einverständlich zwischen Übersetzer 1 und 2 gelöst. Version T12 (ESM3 online) ist somit die Synthese der Versionen T1 und T2 und beruht auf dem Einverständnis von Übersetzer 1 und 2.

\section{Phase 3: Rückübersetzung}

Zwei unabhängige Personen englischer Muttersprache übersetzten Version T12 vom Deutschen ins Englische als Versionen BT1 und BT2 zurück (BT: Back Translation). Beiden Übersetzern waren die original Version sowie Versionen T1 und T2 des Fragebogens unbekannt. Phase 3 wurde mit den Versionen BT1 und BT2 abgeschlossen (ESM 4 und ESM 5 online).

\section{Phase 4: Erstellung der präfinalen Version durch Expertenkomitee}

Ein Expertenkomitee entwickelte die präfinale Fassung des Fragebogens (Tab. 1). Es umfasste den ursprünglichen Verfasser des englischen Fragebogens und zwei Übersetzer, einer mit deutscher, der andere mit englischer Muttersprache. Jedem Mitglied des Komitees wurden alle erstellten Fragebogen-Versionen aus früheren Stadien der Studie (T1, T2, T12, BT1 und BT2), der Originalfragebogen sowie die während der Übersetzungen erstellten Berichte zur Verfügung gestellt. Das Expertenkomitee beurteilte und bestätigte die semantische, idiomatische, konzeptuelle und Erfahrungsäquivalenz zwischen der original und der übersetzten Version des IEQ, was im finalen Bericht (FR) festgehalten ist (ESM6 online). Erfahrungsäquivalenz bedeutete hierbei, dass bei der Übersetzung von Items, mit den Inhalten verbundene Erfahrungen in einer bestimmten Kultur durch ähnliche Erfahrungen in der Zielkultur übersetzt werden. Bei zu wörtlichen Übersetzungen, kann die Bedeutung von Items zu stark divergierenden kulturellen Konstrukten verloren gehen [1]. Nachdem die Äquivalenz aller Versionen vom Expertenkomitee als zufriedenstellend gewertet worden war, begann die Vortestung.

\section{Phase 5: Vortestung}

Die Vortestung des präfinalen deutschsprachigen IEQ-Fragebogens wurde an einer deutschen (Schmerzambulatorium der Universität Saarland, Homburg UKS) und einer schweizerischen (Schmerzsprechstunde des Zentrums für Zahnmedizin der Universität Zürich ZZM) ambulanten Schmerzbehandlungseinrichtung durchgeführt.

\subsection{Stichprobe}

Einundzwanzig Teilnehmende (10 an der UKS und 11 am ZZM) waren einverstanden, die deutsche Version des IEQ auszufüllen und Rückmeldung über dessen Formulierungen und allgemeine Verständlichkeit in einem strukturierten Interview zu geben. Teilnehmende rapportieren Schmerzen als Folge von emotional belastenden Ereignissen, wie Unfälle, Fehlbehandlungen, Komplikationen nach Operationen, Verlust von Beziehungen, Arbeitsfähigkeit oder sozialen Status, was in anderen Studien mit wahrgenommener Ungerechtigkeit in Zusammenhang gebracht wurde $[6,15]$. 
Die Einschlusskriterien waren: Schmerzen unterschiedlicher Ursache, Dauer und Typs, gutes Verständnis der deutschen Sprache und Volljährigkeit. Es wurden auch Teilnehmende eingeschlossen deren Muttersprache nicht Deutsch war, insofern sie über gute umgangssprachliche Deutschkenntnisse verfügten. Die Sprachkompetenz wurde jedoch nicht formal getestet.

Ausgeschlossen wurden Teilnehmende mit kognitiven Defiziten, wie Suchterkrankungen, Psychosen, Demenz oder Schädelhirntraumata. Interessierte Teilnehmende wurden über den Ablauf der IEQVortestung mündlich und schriftlich informiert und unterzeichneten eine Einverständniserklärung zur Studienteilnahme. Diese erfolgte ohne finanzielle Vergütung.

\subsubsection{Interviewer}

Alle Interviews wurden durch zwei klinische Psychologen durchgeführt, die an den oben erwähnten Schmerzbehandlungseinrichtungen arbeiteten (BS, ZZM und KW, UKS).

\subsubsection{Standardisiertes Interview}

Teilnehmende füllten zwischen Juli 2014 und Januar 2015 die präfinale deutsche IEQ-Version in Anwesenheit der Interviewer aus. Das darauffolgende standardisierte Interview orientierte sich an den Empfehlungen für die Vortestung und transkulturelle Anpassungen von Selbstbericht-Fragebögen $[1,16]$. Der Ablauf des Interviews und die Erfassung der Antworten wurden mit Hilfe einer webbasierten Anwendung realisiert. Die Antworten der Teilnehmenden wurden durch die Interviewer zusammengefasst und direkt in einer MySQL Datenbank auf einem kommerziellen Webserver in der Schweiz gespeichert (www.scientific-affairs.ch). Folgende Fragen wurden durch die Interviewer gestellt (Antwortoptionen in Klammern):

Patientenmerkmale: 'Geschlecht', 'Alter', 'Länge der obligatorischen Schulbildung ('6 Jahre' / '9 -10 Jahre'), und 'Art der Berufsausbildung' ('keine' / 'Berufslehre' / 'Matura/Abitur' / 'höhere Berufsausbildung').

Verständlichkeit: Teilnehmende wurden gefragt: 'Gab es Ausdrücke, die nicht verständlich waren?' ( 'ja' / 'nein') [16].

Akzeptanz: Teilnehmende wurden gefragt: 'Gab es Ausdrücke, die inakzeptabel waren?' ( 'ja' / 'nein') [16].

Beleidigung: Teilnehmende wurden gefragt: 'Gab es Ausdrücke, die beleidigend waren?' ( 'ja' / 'nein') [16].

Wurde eine der obigen drei Fragen bejaht, wurde gefragt, auf welche IEQ-Items sich dies bezog. Bei ,nein' folgte die nächste Frage.

Für die Summe der zwölf IEQ-Items wurde ein Maximum von 5\% 'ja'-Antworten als akzeptables Qualitätskriterium für die oben erwähnten Fragen festgelegt. Als zweites Kriterium wurde das Fehlen eines signifikanten Unterschiedes der Häufigkeit von 'ja'-Antworten zwischen den zwölf IEQ-Items definiert.

Bedeutung der IEQ-items und Überlegungen zur gewählten Antwortoption: Zu jedem der zwölf IEQItems wurden gemäss Protokoll zur transkulturellen Adaptation von Selbstbericht Fragebogen folgende zwei Fragen gestellt [1]:

a) Worum geht es bei dieser Aussage?

b) Welche Überlegungen führten zur gewählten Antwort?

\subsubsection{Bewertung}


Alle Antworten zur Bedeutung der zwölf IEQ-Items und die Überlegungen zur gewählten Antwortoption wurden durch zwei unabhängige Mitarbeitende der Studie bewertet, die nicht an den Interviews des Pretests beteiligt waren (PN, Deutsches Rotes Kreuz Schmerzzentrum Mainz (DRK) und SH, Schmerzambulanz des Kantonsspitals St. Gallen (KSG)). Dazu beantworteten die Rater folgende zwei Fragen mit "ja" oder "nein":

1) "Wurde die die Bedeutung der Aussage durch den Teilnehmenden verstanden?" und

2)“Ist die Begründung der gewählten Antwortoption plausibel?”.

Für die Analyse der Beurteilungen wurden "ja"-Antworten der Wert 1 und "nein"-Antworten der Wert 0 zugeordnet. Die beiden Beurteiler erhielten weder zusätzliche Informationen, wie die Bedeutung der Aussagen, noch die wie Plausibilität der gewählten Antwortoptionen zu interpretieren war.

Wie für die Bewertung durch die Teilnehmenden, wurde für die Bewertungen durch die Rater die Summe der zwölf IEQ-Items ein Maximum von 5\% 'nein'-Antworten als akzeptables Qualitätskriterium für die oben erwähnten Fragen festgelegt. Als zweites Kriterium wurde das Fehlen eines signifikanten Unterschiedes der Häufigkeit von 'nein'-Antworten zwischen den zwölf IEQ-Items definiert.

\subsubsection{Datenanalyse}

Die Daten wurden mittels SPSS (IBM, Version 23) analysiert. Für die Beschreibung der Stichprobe und die Häufigkeit und Verteilung der gewählten Antwortoptionen wurden deskriptive Statistiken benutzt. Der Kruskal-Wallis Test wurde für den Vergleich der Häufigkeit von "ja" und "nein"-Antworten zwischen den Items genutzt. Um die Verteilung der gewählten Antwortoptionen auf Normalität zu prüfen wurde der Kolmogorow-Smirnov-Test verwendet. Die Stärke der Übereinstimmung der beiden Rater in der Bewertung des Verständnisses der Bedeutung der Aussagen, sowie für die Bewertung der Plausibilität der gewählten Antwortoption (s. 2.1.4) wurde mit Cohen's Kappa berechnet. Das Signifikanzniveau wurde für alle verwendeten statistischen Verfahren auf $p \leq 0.05$ festgelegt.

\subsubsection{Ethik}

Diese Studie wurde durch die Ethikkommission des Kantons Zürich, Schweiz (KEK-ZH-Nr. 20140066) und der Ärztekammer des Saarlandes, Saarbrücken, Deutschland (Nr. 50/14) bewilligt.

\subsection{Resultate}

Die Daten zweier der 21 Teilnehmenden wurden nicht in die Auswertung aufgenommen, da diese trotz guter umgangssprachlicher Deutschkenntnisse bei der Beantwortung des IEQ und der Fragen zur Bedeutung der Items und der Begründung der gewählten Antwortoptionen erhebliche Verständnisschwierigkeiten zeigten, was auf deren Status als Nichtmuttersprachler zurückzuführen war.

\section{Stichprobencharakteristika:}

Die Daten von neunzehn (10 weibliche) Teilnehmenden wurde in dieser Studie ausgewertet. Die Stichprobencharakteristika sind in Tab. 2 dargestellt. Die Teilnehmenden der Vortestung repräsentierten in Bezug auf Geschlecht und Altersverteilung eine erwerbstätige Population in Deutschland und der Schweiz im Jahre 2016 [9,11].

Beurteilung der Verständlichkeit, Akzeptanz, Beleidigung der IEQ-Items durch die Teilnehmenden:

Die IEQ Items wurden auf Verständlichkeit und Akzeptanz der Teilnehmenden untersucht. Außerdem wurde geprüft, dass keine der Items als beleidigend empfunden wurde. 
Nur Items 4 und 9 wurden jeweils durch einen der 19 Teilnehmenden als "schwierig zu verstehen" bewertet (Tab. 3). Dies entspricht $0.9 \%$ von 228 möglichen Antworten (12 Items bei 19

Teilnehmenden) und liegt somit unter der $5 \%$ Grenze. Es gab keinen signifikanten Unterschied in der Häufigkeit von Bewertungen als 'schwer verständlich' zwischen den Items (Kruskal-Wallis: $X^{2}=10.04$, $\mathrm{df}=11, p=0.53$ ). Keines der 12 Items wurde als inakzeptabel oder beleidigend beurteilt.

Bewertung der Bedeutung der IEQ-Items:

Anhand der Interviews klassifizierte Rater 1 (R1) sechs von 228 (2.6\%) Bewertungen und Rater 2 (R2) neun von 228 Bewertungen (4.0\%) als 'nicht verstanden' (kombiniert 15 von 456, 3.3\%, s. Tab. 4). Ein Maximum von 3 Nennungen fand sich jeweils bei Item 3 und Item 9 . Es wurde kein signifikanter Unterschied zwischen den Items in Bezug auf die Häufigkeit von Bewertungen 'Bedeutung nicht verstanden' zwischen allen Items gefunden (Kruskal-Wallis: $\mathrm{X}^{2}=13.41, \mathrm{df}=11, p=$ 0.27).

Die Interrater-Reliabilität zwischen R1 und R2 für die Bewertung des Verständnisses der Items war überraschend tief und nicht signifikant (Cohen's Kappa: $\mathrm{k}=0.11 p=0.11$ ).

\section{Bewertung der Plausibilität der gewählten Antwortoption:}

Rater 1 bewertete fünf von 228 (2.19\%) und R2 vier von 228 (1.75\%) Antwortoptionen als "nicht plausibel' (R1 und R2 kombiniert neun von 456, 1.97\%, s. Tab. 5). Die gewählten Antwortoptionen von Item 3 und Item 9 wurden erneut am häufigsten als 'nicht plausibel' bewertet. Es fand sich kein signifikanter Unterschied in der Häufigkeit der Bewertung 'Antwort nicht plausibel' zwischen den IEQItems (Kruskal-Wallis: $\mathrm{X}^{2}=13.91, \mathrm{df}=11, p=0.24$ ).

Die Interrater-Reliabilität zwischen R1 und R2 war erneut sehr tief und ohne signifikante Korrelation (Cohen's Kappa: $\mathrm{k}=-0.02 p=0.76$ ).

\section{Häufigkeit und Verteilung der gewählten Antwortoptionen:}

Die Häufigkeit der gewählten Antwortoptionen ist in Abb. 1 dargestellt.

Die Verteilung der gewählten Antwortoptionen ist leicht linksschief, flachgipflig und entspricht keiner Normalverteilung (Tab. 6).

\section{Diskussion}

Wahrgenommene Ungerechtigkeit entsteht meistens in Situationen in denen Verluste oder Verletzungen auftreten, die auf Fehler oder Unachtsamkeiten anderer zurückzuführen sind. Es ist wichtig, diese Wahrnehmungen akkurat und verlässlich zu erkennen und beurteilen zu können, da häufige Wahrnehmungen von Ungerechtigkeit negative Einflüsse auf körperliches und seelisches Wohlbefinden, sowie auf den Heilungsprozess nach Verletzungen haben können [12].

Um Wahrnehmungen von Ungerechtigkeit auch bei deutschsprachigen Patienten verlässlich einschätzen zu können, wurde der IEQ in die deutsche Sprache übersetzt, kulturell angepasst und die erstellte Version in Bezug auf Verständlichkeit und Präzision analysiert. Diese Studie bestätigte die Verständlichkeit und Präzision der deutschen Übersetzung des IEQ.

Alle IEQ-Items erfüllten die zuvor definierten Qualitätskriterien. Die Teilnehmenden beurteilten keines der IEQ-Items als inakzeptabel oder beleidigend. Nur IEQ-Items 4 und 9 wurden je einmal als schwer verständlich beurteilt, was aber unterhalb des $5 \%$ Grenzwertes lag. Außerdem schätzten beide Rater weniger als $5 \%$ der Erklärungen der Teilnehmenden als 'Bedeutung nicht verstanden' oder 'gewählte Antwortoption nicht plausibel' ein. Nur für die IEQ-Items 3 und 9 fanden sich leicht häufigere kritische Bewertungen in Bezug auf die verstandene Bedeutung wie auch für die Plausibilität der gewählten Antwortoptionen. Diese blieben aber erneut unterhalb der festgelegten Qualitätskriterien. Bei IEQ- 
Item 3 könnte diese Auffälligkeit durch die Übersetzung von 'negligence' mit 'Unachtsamkeit' erklärt werden, welche möglicherweise nicht von allen Teilnehmenden gut verstanden wurde. In der finalen Version wurde bei Item 3 deshalb "Unachtsamkeit" durch "Unaufmerksamkeit" ersetzt. Dies war die einzige textliche Änderung des Fragebogens. Bei IEQ-item 9 konnte keine plausible Erklärung für die kritischen Bewertungen gefunden werden. Andererseits sollten diese Abweichungen nicht überbewertet werden, da sie nicht signifikant von der Bewertung anderer Items abwichen, die Qualitätskriterien eingehalten wurden und die Interrater-Reliabilität erstaunlich tief war. Da die Items den definierten Qualitätskriterien entsprachen und die oben erwähnte sprachliche Änderung als synonym betrachtet werden kann, wurde nach der sprachlichen Änderung von Item 3 keine zweite Vortestung durchgeführt.

Zu der tiefen Übereinstimmung zwischen den Ratern hat vermutlich auch der Umstand beigetragen, dass für die Bewertung der erklärten Bedeutung der Antworten und der Plausibilität der gewählten Antwortoptionen kein standardisiertes Verfahren verwendet wurde. Beide Rater erhielten abgesehen von der Versuchsinstruktion keinerlei Hinweise, wie die Antworten interpretiert oder bewertet werden sollten. Außerdem standen beiden Ratern lediglich Transkripte der zusammengefassten Antworten der Teilnehmenden zur Beurteilung zur Verfügung. Ton- oder Videoaufnahmen hätten vermutlich weitere nicht-verbale Informationen beinhalten können, die für die Interpretationen der Antworten hilfreich hätten sein können. Somit sind die Definitionen von Kriterien wie die von Teilnehmenden erklärte Bedeutung von Items und die Plausibilität von gewählten Antwortoptionen standardisiert bewertet werden könnten, ein wichtiger Schritt für die zukünftige Forschung auf dem Gebiet transkultureller Übersetzungen.

Die Häufigkeitswerte der gewählten Antwortoptionen waren nicht normal verteilt. Der Umstand, dass die Verteilung linksschief und flachgipflig war, kann mit der Auswahl und geringen Anzahl der Teilnehmenden erklärt werden, die bewusst nach Erfahrungen von wahrgenommener Ungerechtigkeit in der Lebensgeschichte ausgewählt wurden.

Die Daten von zwei fremdsprachigen Teilnehmenden mit guter umgangssprachlicher Deutschkompetenz wurden nicht in die Auswertung einbezogen. Diese zeigten beim Ausfüllen des $I E Q$, sowie bei den Fragen nach der Bedeutung der Items und der Begründung der gewählten Antwortoptionen erhebliche Schwierigkeiten. Dies zeigt, dass hohe Sprachkompetenz eine wesentliche Voraussetzung zur kompetenten Beantwortung anspruchsvoller psychologischer Konstrukte wie wahrgenommene Ungerechtigkeit ist. Der Einsatz des IEQ als Screeninginstrument bei fremdsprachigen Patienten sollte deshalb zurückhaltend erfolgen und für die Interpretation der Ergebnisse zusätzliche Informationsquellen einbezogen werden. Bei zukünftigen Untersuchungen sollte eine formale Beurteilung der sprachlichen Kompetenz einer Teilnahme vorausgehen.

Um eine möglichst originalgetreue Übersetzung zu ermöglichen, wurde entschieden die deutschen Formulierungen der Items möglichst nahe an den englischen Originalen zu halten. Dies kann unter Umständen bei einzelnen Items zu stilistisch ungewohnten Formulierungen führen. Dies bedeutet allerdings keine verminderte Präzision oder Verständlichkeit der Items, wie diese Studie feststellte, und kann deshalb als kosmetischer Makel angesehen werden.

Letztlich war die Stichprobengröße von 19 Teilnehmenden vergleichsweise gering. Allerdings ist diese Studie lediglich als Vorstufe zur Validierung anzusehen, welche eine weitaus größere Stichprobengröße aufwies.

\section{Fazit für die Praxis}

Die deutsche Übersetzung des IEQ zeigte für die meisten Teilnehmenden ein hohes Maß an Verständlichkeit und die Inhalte der Items wurden mit hoher Plausibilität erkannt. Von daher 
sind für die Verwendung des Fragebogens für die Validierungsstudie keine wesentlichen Änderungen notwendig.

\section{Interessenkonflikt}

Die Autoren bestätigen, dass hinsichtlich der Veröffentlichung dieser Studie keine Interessenkonflikte bestehen.

Die deutsche Version des IEQ wird unter folgender Adresse verfügbar sein: http://sullivanpainresearch.mcgill.ca/ieq.php

\section{Verzeichnis der Tabellen und Abbildungen mit Legenden}

Abb. 1: Häufigkeitsverteilung der gewählten Antwortoptionen.

Tab. 1: Präfinale Version des IEQ

Tab. 2: Stichprobencharakteristika: Aufgrund von Mehrfachnennungen beträgt die Anzahl Nennungen bei der Berufsausbildung 27, die prozentualen Werte beziehen sich auf diesen Wert.

Tab. 3: Häufigkeit von bejahenden Antworten durch die Teilnehmenden $(N=19)$ zu folgenden drei Fragen: Gab es IEQ-Items, die a) schwierig zu verstehen, b) inakzeptabel, c) oder beleidigend waren?

Tab. 4: Häufigkeit der Bewertungen "Bedeutung nicht verstanden" durch Rater 1 (R1) und 2 (R2) gruppiert nach Item.

Tab. 5: Häufigkeit der Bewertungen "Antwort nicht plausibel" durch Rater 1 (R1) und 2 (R2) gruppiert nach Item.

Tab. 6: Verteilung der gewählten Antwortoptionen und Überprüfung der Normalverteilung

\section{Literatur}

[1] Beaton DE, Bombardier C, Guillemin F, Ferraz MB. Guidelines for the process of cross-cultural adaptation of self-report measures. Spine 2000;25(24):3186-91.

[2] Fetchenhauer D, Huang $X$. Justice sensitivity and distributive decisions in experimental games. Personality and Individual Differences 2004;36(5):1015-29.

[3] Gatchel RJ, Adams L, Polatin PB, Kishino ND. Secondary loss and pain-associated disability: theoretical overview and treatment implications. Journal of occupational rehabilitation 2002;12(2):99-110.

[4] Hafer CL, Bègue L. Experimental research on just-world theory: problems, developments, and future challenges. Psychological bulletin 2005;131(1):128-67. 
[5] La Cour P, Smith AA, Schultz R. Validation of the Danish language Injustice Experience Questionnaire. Journal of health psychology 2017;22(7):825-33.

[6] Miller DT. Disrespect and the experience of injustice. Annual review of psychology 2001;52:52753.

[7] Mohiyeddini C, Schmitt MJ. Sensitivity to befallen injustice and reactions to unfair treatment in a laboratory situation. Social Justice Research 1997;10(3):333-53.

[8] Rodero B, Luciano JV, Montero-Marín J, Casanueva B, Palacin JC, Gili M, López del Hoyo Y, Serrano-Blanco A, Garcia-Campayo J. Perceived injustice in fibromyalgia: Psychometric characteristics of the Injustice Experience Questionnaire and relationship with pain catastrophising and pain acceptance. Journal of psychosomatic research 2012;73(2):86-91.

[9] Schweizerische Eidgenossenschaft, Bundesamt für Statistik. Die Bevölkerung der Schweiz. Available at: https://www.bfs.admin.ch/bfs/de/home/statistiken/bevoelkerung.html. Accessed 16 April 2018.

[10] Scott W, Trost Z, Milioto M, Sullivan MJL. Further validation of a measure of injury-related injustice perceptions to identify risk for occupational disability: a prospective study of individuals with whiplash injury. Journal of occupational rehabilitation 2013;23(4):557-65.

[11] Statistisches Bundesamt. Bevölkerungsstand. Available at: https://www.destatis.de/DE/ZahlenFakten/GesellschaftStaat/Bevoelkerung/Bevoelkerung.html. Accessed 16 April 2018.

[12] Sullivan MJL, Adams H, Horan S, Maher D, Boland D, Gross R. The role of perceived injustice in the experience of chronic pain and disability: scale development and validation. Journal of occupational rehabilitation 2008;18(3):249-61.

[13] Sullivan MJL, Thibault P, Simmonds MJ, Milioto M, Cantin A-P, Velly AM. Pain, perceived injustice and the persistence of post-traumatic stress symptoms during the course of rehabilitation for whiplash injuries. Pain 2009;145(3):325-31.

[14] van Leeuwen WF, van der Vliet QMJ, Janssen SJ, Heng M, Ring D, Vranceanu A-M. Does perceived injustice correlate with pain intensity and disability in orthopaedic trauma patients? Injury 2016;47(6):1212-6.

[15] Wallin MKM, Raak RI. Quality of life in subgroups of individuals with whiplash-associated disorders. European journal of pain (London, England) 2008;12(7):842-9.

[16] World Health Organisation WHO. Process of translation and adaptation of instruments. Available at: http://www.who.int/substance_abuse/research_tools/translation/en/index.html. Accessed 17 August 2017.

[17] Yakobov E. Validation of the Injustice Experiences Questionnaire Adapted for Use with Patients with Severe Osteoarthritis of the Knee. J Arthritis 2014;03(02).

[18] Yamada K, Adachi T, Mibu A, Nishigami T, Motoyama Y, Uematsu H, Matsuda Y, Sato H, Hayashi K, Cui R, Takao Y, Shibata M, Iso H. Injustice Experience Questionnaire, Japanese Version: Cross-Cultural Factor-Structure Comparison and Demographics Associated with Perceived Injustice. PloS one 2016;11(8):e0160567. 